\title{
Sobrecarga y calidad de vida de cuidadores de personas con cáncer en cuidados paliativos
}

Overburden and Quality of Life of Caregivers of Patients with Cancer during Palliative Care

Sobrecarga e qualidade de vida de cuidadores de pessoas com câncer em cuidados paliativos

Como citar este artículo:

Arias-Rojas Mauricio, Carreño Moreno Sonia, Sepúlveda García Aura, Romero Ballesteros Ingrith. Sobrecarga y calidad de vida de cuidadores de personas con cáncer en cuidados paliativos. Revista Cuidarte. 2021;12(2):e1248.

http://dx.doi.org/10.15649/cuidarte.1248

Revista Cuidarte

Rev Cuid. May - Ago 2021; 12(2): e1248

doij http://dx.doi.org/10.15649/cuidarte.1248

E-ISSN: $2346-3414$

(1) Mauricio Arias-Rojas'

(1) Sonia Carreño Moreno²

(1) Aura Sepúlveda García ${ }^{3}$

(1) Ingrith Romero Ballesteros ${ }^{4}$

1 Profesor asistente, Facultad de Enfermería, Universidad de Antioquia. Grupo de investigación práctica de enfermería en el contexto social GIPECS. Medellín, Colombia.

E-mail: emauricio.arias@udea.edu.co Autor de correspondencia

2 Profesora asociada, Facultad de Enfermería, Universidad Nacional de Colombia, Bogotá, Colombia.

E-mail: spcarrenom@unal.edu.co

3 Enfermera, Auxiliar de investigación, Facultad de Enfermería, Universidad de Antioquia.

E-mail: aurasepulveda08@gmail.com

4 Enfermera, Auxiliar de investigación, Facultad de Enfermería, Universidad de Antioquia.

E-mail: iyromerob@unal.edu.co

\section{Resumen}

Introducción. El cuidador familiar experimenta en muchos casos sobrecarga relacionada con asumir el cuidado de una persona, esto a su vez puede tener implicaciones para su calidad de vida. El objetivo de este estudio fue describir las asociaciones entre el nivel de sobrecarga del cuidado y la calidad de vida de cuidadores de pacientes con cáncer en cuidados paliativos que asistían a una institución de IV nivel de la ciudad de Medellín, Colombia. Materiales y métodos. Estudio descriptivo correlacional de corte transversal desarrollado con 62 cuidadores familiares. Se aplicaron los cuestionarios de caracterización del participante, escala de sobrecarga del cuidador Zarit y calidad de vida del cuidador. Se realizaron pruebas de correlación por coeficiente de Pearson y modelo de regresión simple. Resultados. Se encontró un coeficiente de Pearson de $r=-0.54$ con una $p<0.0001$ entre la sobrecarga y la calidad de vida; surgieron además correlaciones negativas entre todas dimensiones de calidad de vida y la sobrecarga del cuidado. Se estableció un modelo de regresión en el que la sobrecarga del cuidador ( $\beta=-0.556$ IC 95\% $=-3.114$ - -1.237) y el ser cuidador casado o en unión libre $(\beta=0.258$ IC $95 \%=0.907-52.99)$ son variables predictoras de la calidad de vida. Conclusiones. Existe una correlación inversa y fuerte entre la sobrecarga y la calidad de vida del cuidador familiar de la persona con cáncer en cuidados paliativos.

Palabras clave: Costo de Enfermedad; Calidad deVida; Cuidadores; Cuidados Paliativos; Cáncer.

Recibido: mayo 13 de 2020 Aceptado: diciembre 17 de 2020

Publicado: abril 30 de 2021 $\square *$ Correspondencia Mauricio Arias-Rojas

E-mail:emauricio.arias@udea.edu.co 


\section{Overburden and Quality of Life of Caregivers of Patients with Cancer during Palliative Care}

\section{Abstract}

Introduction: Family caregivers often experience caregiving overburden, which in turn may have implications for their quality of life. This study is aimed at describing the associations between the level of caregiving overburden and the quality of life of caregivers of patients with cancer during palliative care attending a quaternary healthcare institution in Medellin, Colombia. Materials and Methods: A cross-sectional descriptive correlational study was conducted with 62 family caregivers. Participants' characterization, Zarit Caregiver Burden Scale and Caregiver Quality of Life questionnaires were administered. Correlation tests were performed using Pearson's correlation coefficient and simple linear regression model. Results: A Pearson's correlation coefficient of $r=-0.54$ at $p<0.0001$ was found between caregiver overburden and quality of life. Negative correlations were also identified across all dimensions of overburden and quality of life in caregivers. A regression model was established in which caregiver overburden $(\beta=-0.556 \mathrm{Cl} 95 \%=-3.114--1.237)$ and being a married or common-law partnered caregiver $(\beta=0.258$ IC $95 \%=0.907-52.99)$ are predicting variables of quality of life. Conclusions: A strong inverse correlation was found between overburden and quality of life of family caregivers of patients with cancer during palliative care.

Key words: Cost of Illness; Quality of Life; Caregivers; Palliative Care; Cancer.

\section{Sobrecarga e qualidade de vida de cuidadores de pessoas com câncer em cuidados paliativos}

\section{Resumo}

Introdução. O cuidador familiar vivencia, em muitos casos, uma sobrecarga relacionada ao cuidado de uma pessoa, isso por sua vez pode ter implicações na sua qualidade de vida. $O$ objetivo deste estudo foi descrever as associações entre o nível de sobrecarga assistencial e a qualidade de vida de cuidadores de pacientes com câncer em cuidados paliativos atendidos em uma instituição de IV nível na cidade de Medellín, Colômbia. Materiais e métodos. Estudo descritivo correlacionado com a seção transversal desenvolvida com 62 cuidadores familiares. Foram aplicados questionários de caracterização dos participantes, escala de sobrecarga do cuidador Zarit e qualidade de vida do cuidador. Os testes de correlação foram realizados por meio do coeficiente de Pearson e modelo de regressão simples. Resultados. Encontrou-se coeficiente de Pearson de $r=-0.54$ com uma $p<0.0001$ entre sobrecarga e qualidade de vida; surgiram também correlações negativas entre todas as dimensões da qualidade de vida e a sobrecarga de cuidados. Um modelo de regressão foi estabelecido no qual a sobrecarga do cuidador ( $\beta=-0.556$ IC $95 \%=-3.114--1.237$ ) e o ser cuidador casado ou em união estável ( $\beta$ $=0.258$ IC $95 \%=0.907-52.99)$ são variáveis preditivas de qualidade de vida. Conclusões. Existe uma correlação inversa e forte entre a sobrecarga e a qualidade de vida do cuidador familiar da pessoa com câncer em cuidados paliativos.Conclusões. Existe uma correlação forte e inversa entre a carga e a qualidade de vida do cuidador familiar da pessoa com câncer em cuidados paliativos.

Palavras chave: Efeitos Psicossociais da Doença; Qualidade de Vida; Cuidadores; Cuidados Paliativos; Câncer. 


\section{Introducción}

En países de ingresos medios como Colombia, el cáncer representa la segunda causa de mortalidad en la población a pesar de que el avance en los tratamientos la ha posicionado en el mundo como una enfermedad crónica'. Esto lleva a la necesidad de implementar atención de cuidados paliativos por diferentes motivos como estadios avanzados de la enfermedad o fallos en el tratamiento ${ }^{2}$; sin embargo, el estudio de Pastrana et al. ${ }^{3}$ indican que Colombia tiene 15.5 servicios de cuidados paliativos menos que Suecia y 7.5 servicios menos que Chile y consume 6.4 miligramos de morfina por habitante por año menos que Argentina, indicadores que aunados a la falta de formación de recurso humano en el área y falta de reglamentación de la normativa en cuidados paliativos, hacen que el país se encuentre en desventaja con países de ingresos similares y muy por debajo de países con ingresos superiores.

La atención en cuidados paliativos es una experiencia donde existen dos actores principales. El paciente que experimenta el cáncer avanzado y su cuidador familiar ${ }^{4}$ quien asume la responsabilidad del cuidado al paciente. En general, esta vivencia se caracteriza por ser compleja y con grandes necesidades desde lo físico, lo emocional y lo espiritual, y en muchos de estos casos el cuidador debe asumir este rol sin una adecuada preparación, acompañamiento continuo de los profesionales de la salud y redes de apoyo apropiadas, lo que genera en este una percepción de baja calidad de vida por los múltiples cambios a los que se debe enfrentar ${ }^{5}$.

Diferentes estudios ${ }^{6,7}$ sobre la calidad de vida en cuidadores familiares de personas en cuidados paliativos han identificado que esta es menor que en otras poblaciones. Por ejemplo, en cuidadores de pacientes en tratamiento activo contra el cáncer, siendo la dimensión psicológica la que tiene peores niveles de calidad de vida,

Diferentes estudios ${ }^{6,7}$ sobre la calidad de vida en cuidadores familiares de personas en cuidados paliativos han identificado que esta es menor que en otras poblaciones lo que está relacionado con las formas de afrontamiento de la situación, la poca satisfacción con el soporte social recibido y el conocimiento sobre la progresión de la enfermedad y el final de la vida. Esto a su vez, genera en los cuidadores tristeza, dolor, agotamiento, ansiedad y problemas para dormir, desarrollando angustia psicosocial, la cual conlleva a que casi el $74 \%$ de ellos presente depresión según un estudio ${ }^{8}$.

Con respecto a la percepción de sobrecarga del cuidado, algunas investigaciones han mostrado que desde la experiencia de estos cuidadores existe una carga enfocada en el agotamiento físico, una carga mental relacionada con sentimientos de pérdida de autoestima, desesperanza, desánimo, tristeza y soledad, y carga social frente al rol femenino en el cuidado y la alteración de la dinámica familiar ${ }^{9}$. En este mismo sentido, los cuidadores refieren que sus cargas más frecuentes corresponden a la sensación de que son las únicas personas de quien depende su familiar, haciendo que se perciba una mayor insatisfacción con su rol dentro del cuidado paliativo ${ }^{10}$.

La relación entre la sobrecarga del cuidado y la calidad de vida de los cuidadores ha sido estudiada en otros contextos, de esta manera, en el estudio de Perpiña et al. ${ }^{11}$, encontraron que los cuidadores de personas en cuidado paliativo presentaban en un $48.1 \%$ ansiedad y el $18.2 \%$ depresión, además de reportar niveles bajos de calidad de vida en la dimensión física. El $63.7 \%$ mostró una sobrecarga moderada en contraste con el $41.6 \%$ que reportaron sobrecarga intensa. Al final, encontraron asociaciones entre la ansiedad, la depresión, la fatiga con la sobrecarga del cuidador $(r=0.65 p<0.001 ; r=0.70 p<0.001$ y $r=0.56 p<0.001$ respectivamente); 
sin embargo, la calidad de vida y sus dimensiones no se correlacionaron de manera significativa con la sobrecarga o las demás variables del estudio.

De igual manera, otro estudio sobre la calidad de vida del cuidador de la persona en cuidado paliativo y su asociación con variables sociodemográficas, evidenciaron que la edad del cuidador se relacionaban con las variables de calidad de vida denominadas Rol físico $(r=0.56 p<0.001)$, y Vitalidad $(r=0.47 p<0.001)$, concluyendo que, independiente del tipo de patología y del tiempo que lleve en el rol, la edad de los cuidadores es un factor importante para la afectación de la calidad de vida, de allí que persiste una percepción más baja de calidad de vida en cuidadores mayores de 60 años, lo que determina la aparición del denominado síndrome del cuidador en el que aparecen tanto síntomas físicos como psícológicos ${ }^{12}$. La sobrecarga, aunque no fue una variable medida, también se atribuye como una de las causas de cansancio del rol ${ }^{13}$.

De acuerdo con lo argumentado, para los profesionales de enfermería es importante investigar sobre este fenómeno, dado que son estos los encargados extender el cuidado del paciente hacia su red proximal, en este caso su cuidador familiar, e identificar también sus necesidades de cuidado derivadas de ejercer este rol. Además, investigar sobre los factores que se relacionan con la calidad de vida del cuidador servirá para generar evidencia en el desarrollo de terapéuticas encaminadas a modificar o controlar dichos factores. Siendo así, el objetivo de este estudio fue describir las asociaciones entre las características y perfil del cuidador y el nivel de sobrecarga del cuidado con la calidad de vida de cuidadores de pacientes con cáncer en cuidados paliativos que asistían a una institución de IV nivel de atención de la ciudad de Medellín, Colombia.

\section{Material y Métodos}

Estudio cuantitativo de diseño descriptivo correlacional de corte transversal ${ }^{14}$. La muestra estuvo conformada por el total de los cuidadores de personas con cáncer en cuidado paliativo que se encontraran hospitalizadas al momento de la recolección de datos, entre mayo y diciembre de 2019. Se invitaron a participar en el estudio a 83 cuidadores de los cuales 62 aceptaron participar. El tamaño muestral fue adecuado para los propósitos del estudio dado que considerando un cálculo de tamaño de muestra a través del programa G-Power con los parámetros de ingreso: F test, Regresión lineal, tamaño del efecto 0.3, probabilidad de error tipo 1 de 0.05, poder (1 beta) 0.95 y total de predictores 10, se obtuvo un tamaño de muestra de 56 participantes.

La muestra se seleccionó a través de un muestreointencional, seincluyeron aquellos participantes que fueran el cuidador familiar principal del paciente con cáncer avanzado hospitalizado en cuidados paliativos, mayores de 18 años y con el test mental SPMSQ menor a 2 errores. Se excluyeron a aquellos cuidadores contratados para cuidar al paciente.

Para la medición de las variables del estudio se aplicaron 3 instrumentos:

- Caracterización del cuidador: se empleó la encuesta de caracterización de la diada (GCPCUN-D), diseñado por el Grupo de Investigación Cuidado de Enfermería al Paciente Crónico de la Facultad de Enfermería de la Universidad Nacional de Colombia. Esta encuesta tiene 23 ítems en los que se identifican características sociodemográficas del cuidador y de la persona enferma, tiempo que lleva como cuidador, las horas diarias dedicadas a cuidar, si es único cuidador y la satisfacción con el apoyo recibido. La escala presentó una comprensibilidad del $95 \%$ y una validez aparente del $100 \%$ en un estudio en Colombia ${ }^{15}$.

- Sobrecarga del cuidado: se aplicó la Entrevista de Percepción de Carga del Cuidado de Zarit, que mide en forma multidimensional el nivel de sobrecarga percibida por el cuidador 
en su rol. El instrumento cuenta con tres dimensiones Impacto (12 ítems), Interpersonal (6 ítems), Competencia y expectativas (4 ítems). Consta de 22 ítems con escala tipo Likert de 5 opciones. La puntuación va desde 22 a 110 puntos, si puntúa $\leq 46$ indica ausencia de sobrecarga, entre 47 y 55 es sobrecarga ligera y $\geq 56$ es sobrecarga intensa. Este instrumento cuenta con pruebas psicométricas en Colombia, con una validez de constructo por rotación varimax que explica el $68 \%$ de la varianza y una confiabilidad con alfa de Cronbach de $0.88^{16,17}$.

- Calidad de vida del cuidador: se aplicó la escala de calidad de vida versión cuidador familiar del paciente con cáncer, diseñada por Betty Ferrell. La escala consta de 37 ítems, que se califican de 0 a 10, donde cero es el peor resultado y diez es el mejor. La escala cuenta con cuatro dimensiones que son bienestar físico (5 ítems), bienestar psicológico (16 ítems), preocupaciones sociales ( 9 ítems) y bienestar espiritual (7 ítems). Su validación en Colombia se ajustó a una estructura de cuatro factores que concuerdan con las dimensiones planteadas en la versión original y una confiabilidad por alfa de Cronbach de $0.86^{18}$.

Los cuestionarios fueron aplicados por dos auxiliares de investigación debidamente entrenadas, quienes obtuvieron el consentimiento informado escrito por parte de los cuidadores familiares previo a la administración de los instrumentos. La información recolectada fue tabulada en una base de datos del programa Microsoft Excel, los análisis estadísticos se realizaron en el programa SPSS v.24, licenciado para la Universidad de Antioquia.

Frente al análisis de los datos, se empleó estadística descriptiva e inferencial. Para la caracterización de los participantes, sobrecarga del cuidado y calidad de vida, se calcularon proporciones, medias, desviaciones estándar e intervalos de confianza del 95\%. La distribución de las variables fue normal de acuerdo con el test de Kolgomorov Smirnov, por tanto, para la exploración de correlaciones se usó el coeficiente de Pearson ( $r$ y Chi cuadrado de Pearson para variables dicotómicas. Se reportaron como valores $\mathrm{p}$ significativos aquellos menores a 0.05 y para el caso de la interpretación de las correlaciones obtenidas se fijaron los valores de menores a 0.3 de magnitud débil, entre 0.3 a 0.6 magnitud moderada y mayores a 0.6 magnitud fuerte $^{19}$. Adicionalmente, se realizó una regresión múltiple entre las variables que mostraron una correlación en el análisis anterior y las que se informaron en los estudios como influyentes en la calidad de vida.

Esta investigación contó con la aprobación del comité de ética e investigaciones de la institución de salud donde se desarrolló el estudio (acta no. 08/2018). Además, se tuvo en cuenta lo dispuesto en la Resolución 008430 de 1993 por la cual se establecen las normas científicas, técnicas y administrativas para la investigación en saludo, así como los siete requisitos éticos en la investigación de Ezekiel Emanuel. Todos los participantes firmaron consentimiento informando.

\section{Resultados}

\section{Caracterización de la población}

La Tabla 1 describe que, de los 62 cuidadores participantes, el 82.30\% eran de género femenino, el $75.80 \%$ no eran únicos cuidadores. En el $38.70 \%$ de los casos eran hijos(as) de la persona con cáncer en cuidado paliativo y para el caso del cuidador secundario el 54.83\% era otro familiar consanguíneo como sobrinos, primos o nietos. Frente a las personas con cáncer en cuidado paliativo, su edad promedio fue de 63 años y el total de ellos tenían un cáncer de base junto 
con metástasis a otros órganos, siendo el de mayor prevalencia los relacionados con el sistema gastrointestinal en un $45.16 \%$.

Tabla 1. Características sociodemográficas de la diada cuidador - paciente

\begin{tabular}{|c|c|}
\hline Característica & $\%(n=62)$ \\
\hline \multicolumn{2}{|l|}{ Datos de la persona con cáncer en cuidados paliativos } \\
\hline \multicolumn{2}{|l|}{ Diagnóstico oncológico } \\
\hline Mieloma/Leucemia/Linfomas & $12.90(8)$ \\
\hline Cáncer de estómago/colon/recto & $25.81(16)$ \\
\hline Cáncer de hígado/páncreas/vesícula & $19.35(12)$ \\
\hline Cáncer de mama & $11.29(7)$ \\
\hline Cáncer de pulmón & $11.29(7)$ \\
\hline Cáncer de cérvix/útero & $4.84(3)$ \\
\hline Cáncer de próstata & $6.45(4)$ \\
\hline Otros & $8.06(5)$ \\
\hline \multicolumn{2}{|l|}{ Género } \\
\hline Masculino & $58.10(26)$ \\
\hline Femenino & $41.90(36)$ \\
\hline \multicolumn{2}{|l|}{ Parentesco con el cuidador } \\
\hline Hijo & $38.70(24)$ \\
\hline Conyugue & $32.30(20)$ \\
\hline Nieto & $6.50(4)$ \\
\hline Hermano & $9.70(6)$ \\
\hline Otro familiar & $12.80(8)$ \\
\hline \multicolumn{2}{|l|}{ Datos del cuidador familiar } \\
\hline \multicolumn{2}{|l|}{ Nivel de escolaridad } \\
\hline Primaria & $35.50(22)$ \\
\hline Secundaria & $40.30(25)$ \\
\hline Técnico y/o Profesional & $24.20(15)$ \\
\hline \multicolumn{2}{|l|}{ Estado civil } \\
\hline Casado(a) & $53.20(33)$ \\
\hline Soltero(a) & $29.00(18)$ \\
\hline Unión Libre & $17.70(11)$ \\
\hline \multicolumn{2}{|l|}{ Ocupación } \\
\hline Hogar & $45.20(28)$ \\
\hline Independiente & $27.40(17)$ \\
\hline Empleado & $14.50(9)$ \\
\hline Pensionado & $12.90(8)$ \\
\hline \multicolumn{2}{|l|}{ Religión } \\
\hline Católica & $82.30(51)$ \\
\hline Cristiana & $8.10(5)$ \\
\hline Ninguna & $9.70(6)$ \\
\hline \multicolumn{2}{|l|}{ Nivel de compromiso religioso } \\
\hline Alto & $77.40(48)$ \\
\hline Medio & $14.50(9)$ \\
\hline Bajo & $8.10(5)$ \\
\hline Cuida a la persona desde el inicio de la enfermedad & $93.50(58)$ \\
\hline
\end{tabular}

Fuente: Datos obtenidos del estudio y analizados en SPSS. 2020

Niveles de sobrecarga del cuidado y calidad de vida del cuidador familiar

La tabla 2 describe las características de los participantes en las variables de razón y los puntajes de sobrecarga y calidad de vida del cuidador. Frente a la edad del cuidador, la media fue de 57 años; llevaban en promedio 19 meses en el desarrollo de su rol y cuidaban al paciente en promedio 18 horas al día. La escala de Karnofsky del paciente presentó un puntaje promedio 
de 71.45, indicando un nivel medio de funcionalidad del paciente. Sobre la sobrecarga del cuidado, el cuidador presentó una sobrecarga de ligera a intensa (ausencia de sobrecarga $56.50 \%$; sobrecarga leve $19.40 \%$ y sobrecarga intensa $24.10 \%$ ), con una media en la dimensión de impacto de 27.44, interpersonal de 8.61 y competencias y expectativas de 11.82 puntos. Para la calidad de vida, el cuidador presentó niveles medios en la totalidad de la escala y todas sus dimensiones.

Tabla 2. Valores descriptivos de las variables medidas en el cuidador familiar

\begin{tabular}{lcccc}
\multicolumn{1}{c}{ Variables } & Mínimo & Máximo & Media & $\begin{array}{c}\text { Desviación } \\
\text { estándar }\end{array}$ \\
\hline Características del cuidado* & & & & \\
$\quad$ Edad del cuidador (años) & 20 & 77 & 57.40 & 15.10 \\
Edad del paciente (años) & 24 & 91 & 63.63 & 14.71 \\
Tiempo que lleva como cuidador (meses) & 1 & 144 & 19.87 & 33.36 \\
$\quad$ Número de horas que dedica al cuidado & 5 & 24 & 18.94 & 5.64 \\
Escala de Karnofsky del paciente & 40 & 90 & 71.45 & 15.56 \\
Sobrecarga del cuidado & & & & \\
Impacto & 13 & 47 & 27.44 & 8.18 \\
Interpersonal & 6 & 16 & 8.61 & 2.90 \\
Competencias y expectativas & 4 & 19 & 11.82 & 3.98 \\
Carga Total & 23 & 79 & 57.87 & 12.21 \\
Calidad de vida del cuidador & & & & \\
Bienestar físico & 9 & 48 & 26.50 & 9.64 \\
Bienestar psicológico & 30 & 141 & 73.24 & 21.77 \\
Preocupaciones sociales & 12 & 80 & 49.81 & 18.32 \\
Bienestar espiritual & 5 & 70 & 45.52 & 12.73 \\
Calidad de vida Total & 62 & 292 & 195.06 & 47.79 \\
\hline
\end{tabular}

*Distribución normal de las variables.

Fuente: Datos obtenidos del estudio y analizados en SPSS. 2020
Sobre la sobrecarga del cuidado, el cuidador presentó una sobrecarga de ligera a intensa (ausencia de sobrecarga $56.50 \%$; sobrecarga leve $19.40 \%$ y sobrecarga intensa $24.10 \%$ )

\section{Correlaciones entre el perfil del cuidador familiar, sobrecarga del cuidado y calidad de vida.}

El análisis estadístico por Chi cuadrado reportó una asociación significativa ( $p=0.038$ ) entre las variables dicotómicas de ser único cuidador y presentar sobrecarga, interpretándose que los participantes al no ser únicos cuidadores en su mayoría tienen ausencia de sobrecarga. Por otra parte, el coeficiente de correlación de Pearson entre la sobrecarga del cuidado y la calidad de vida del cuidador fue moderado $(r=-0.54)$ con un valor $p<0.01$.

Encuantoalas dimensiones delasvariables sobrecarga del cuidado y la calidad de vida del cuidador, se encontraron correlaciones entre el nivel total de sobrecarga del cuidado y el bienestar físico, psicológico, espiritual y las preocupaciones sociales. También se correlacionó la dimensión de impacto de la sobrecarga con todas las dimensiones de la calidad de vida. Igualmente, se correlacionaron la dimensión interpersonal de la
En cuanto a las dimensiones de las variables sobrecarga del cuidado y la calidad de vida del cuidador, se encontraron correlaciones entre el nivel total de sobrecarga del cuidado y el bienestar físico, psicológico, espiritual y las preocupaciones sociales. sobrecarga y las dimensiones física, psicológica y social de la calidad de vida con una magnitud de asociación moderada. La Tabla 3 detalla la matriz de las correlaciones encontradas. 


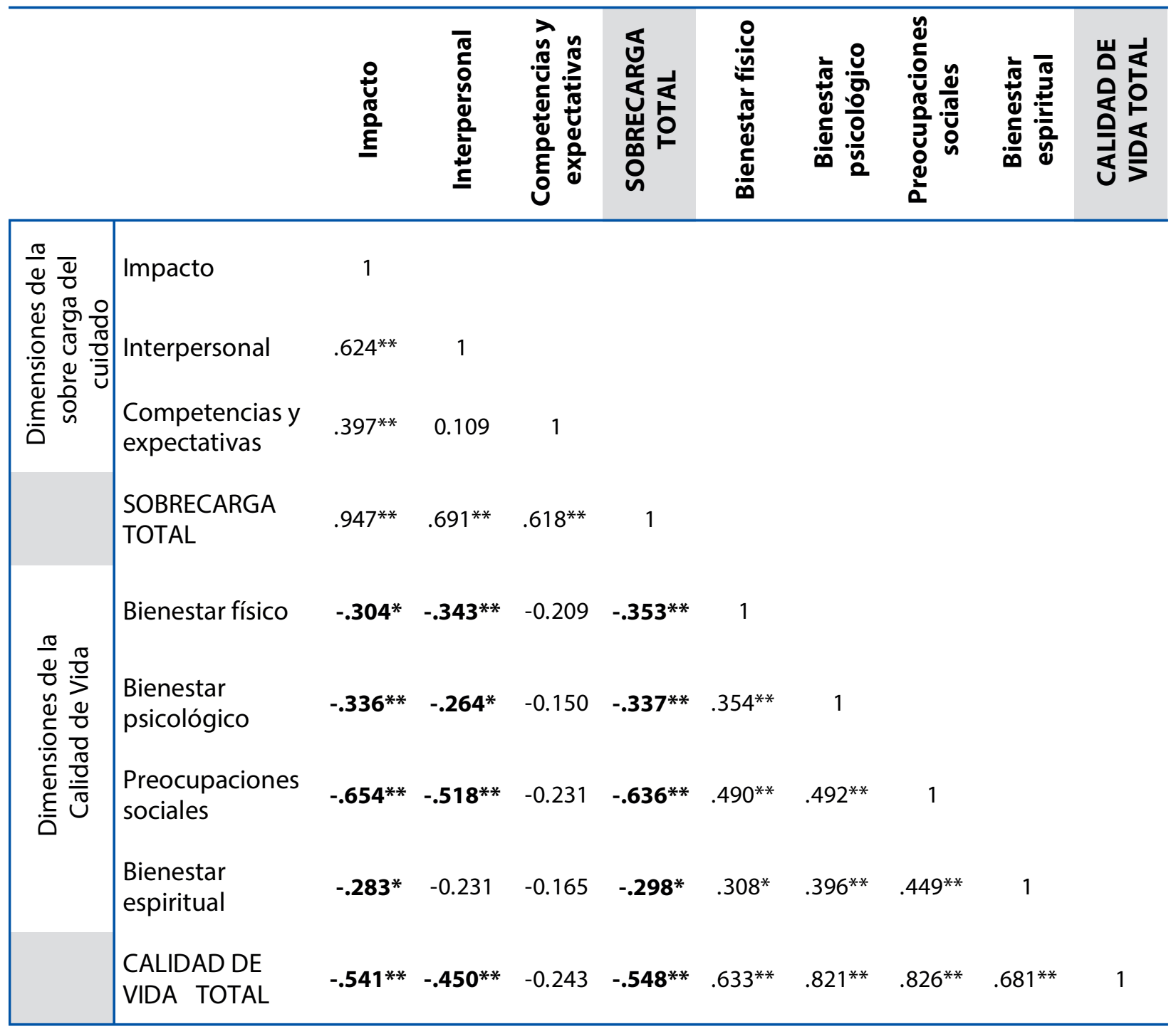

**. La correlación es significativa en el nivel 0,01 (bilateral).

* La correlación es significativa en el nivel 0,05 (bilateral).

Fuente: Datos obtenidos del estudio y analizados en SPSS. 2020

En la Tabla 4, se detalla el modelo de regresión múltiple, el cual se desarrolló entre las variables de caracterización y perfil de cuidado del participante y la sobrecarga del cuidado sobre la calidad de vida del cuidador, considerando esta última como la variable dependiente. Se encontró que, en el modelo derivado, 2 de las 11 variables predictoras representaron el $38 \%$ de la varianza. El estado civil del cuidador familiar ya sea casado o en unión libre, es predictor de la calidad de vida $(t=2.07 \mathrm{p}<0.04)$, así mismo, la sobrecarga del cuidador también es predictora de la calidad de vida $(t=$ El estado civil del cuidador familiar ya sea casado o en unión libre, es predictor de la calidad de vida ( $t=2.07 p<0.04)$, así mismo, la sobrecarga del cuidador también es predictora de la calidad de vida $(t=-4.65 p$ $-4.65 p<0.001)$. $<0.001$ ).

\section{Tabla 4. Modelo de regresión múltiple}




\begin{tabular}{lcc}
\hline \multicolumn{1}{c}{ Variables } & $\boldsymbol{\beta}(\mathbf{I C ~ 9 5 \% )}$ & $\mathbf{p}$ \\
\hline Escala de Karnofsky & $0.086(-0.510 ; 1.036)$ & 0.498 \\
Edad del paciente & $-0.025(-0.970 ; 0.805)$ & 0.853 \\
Edad del cuidador & $0.166(-0.344 ; 1.410)$ & 0.228 \\
Tiempo que lleva como cuidador & $-0.094(-0.496 ; 0.227)$ & 0.458 \\
Número de horas diarias dedicadas al cuidado & $-0.055(-2.625 ; 1.689)$ & 0.665 \\
Nivel total de sobrecarga & $-\mathbf{0 . 5 5 6}(-3.114 ;-1.237)$ & $\mathbf{0 . 0 0 0}$ \\
Género del cuidador & $-0.023(-32.661 ; 26.951)$ & 0.848 \\
Estado civil del cuidador (casado -unión libre) & $\mathbf{0 . 2 5 8}(0.907 ; 52.997)$ & $\mathbf{0 . 0 4 3}$ \\
Cuida a la persona desde el inicio de la enfermedad & $-0.043(-55.648,38.897)$ & 0.723 \\
Compromiso religioso & $0.035(-24.205 ; 32.118)$ & 0.779 \\
Único cuidador & $-0.035(-31.744,23.942)$ & 0.780 \\
\hline
\end{tabular}

$\beta=$ beta; $\mathrm{t}=\mathrm{t}$ de student; $\mathrm{p}=$ nivel de significancia

Fuente: Datos obtenidos del estudio y analizados en SPSS. 2020

\section{Discusión}

Dentro de este estudio, los cuidadores de personas con cáncer en cuidado paliativo son en su mayoría mujeres con un vínculo de parentesco de hijo o conyugue, quienes llevan aproximadamente un año y medio en el rol y que además cuentan con el apoyo de cuidadores secundarios, al declarar que no son únicos cuidadores. Al comparar estas características con otros estudios, se encuentra que son similares al estudio de Rizo et al. ${ }^{20}$, para quienes los cuidadores de personas con estadios avanzados de cáncer, eran $70 \%$ mujeres, 34\% eran cónyuges y el $49 \%$ llevaba entre 1 y 3 años brindando el cuidado a la persona enferma; así mismo, los resultados de Flores et al. ${ }^{21}$ muestran que los cuidadores de adultos mayores tienen en promedio de 36 a 55 años, de sexo femenino (79.7\%), casados (64.4\%) y dedicados al hogar principalmente (50.8\%), dejando en evidencia que el perfil de los participantes del estudio es similar en poblaciones con enfermedades crónicas.

Otro resultado importante está relacionado con el no ser único cuidador, representado en que el $75.8 \%$ de los participantes tenía apoyo de otras personas para las actividades del cuidado, es decir, contaban con un cuidador secundario. Esto puede indicar que su percepción de sobrecarga no es igual que si fuese único cuidador, esta hipótesis es respaldada por Lara et al. ${ }^{22}$ quienes hallaron que el $83.3 \%$ de los cuidadores primarios contaban con cuidadores secundarios, que en su mayoría eran familiares del mismo paciente, lo que les permitía un mejor afrontamiento del nuevo rol como cuidadores. En contraste, otros estudios han demostrado que la familia puede ser una fuente de sobrecarga más que de apoyo para el cuidador, así lo describe Naoki et al. ${ }^{10}$, para quienes la carga del cuidador se correlacionó negativamente con la satisfacción familiar en el cuidado al final de la vida $(r=-0.56 p<0.005)$.

En este mismo sentido, más de la mitad de los cuidadores participantes reportaron ausencia de sobrecarga, lo que puede estar relacionado, no solo con la presencia de otros cuidadores, sino también por la funcionalidad del paciente. Al respecto, por los resultados en la Escala de Karnofsky es probable que estas personas en cuidado paliativo aún no tuvieran cerca del final de la vida y se conservaran independientes para realizar algunas actividades de la vida diaria, 
disminuyendo la percepción de sobrecarga por parte del cuidador $^{23,24}$. No se puede dejar de lado que varios de los participantes refirieron algún grado de sobrecarga y un buen porcentaje la considero como intensa, lo cual puede ser explicado desde la dimensión de impacto del cuidado, debido a que convertirse en cuidador trae cambios mayores en la vida personal, en las dinámicas familiares y laborales llevando a que este experimente que la situación lo supera, tal como lo reportan algunos estudios $^{6,23,25}$.
Al respecto, por los resultados en la Escala de Karnofsky es probable que estas personas en cuidado paliativo aún no tuvieran cerca del final de la vida y se conservaran independientes para realizar algunas actividades de la vida diaria, disminuyendo la percepción de sobrecarga por parte del cuidador ${ }^{23,24}$.

Por otro lado, se ha evidenciado bajos niveles de calidad de vida del cuidador de personas en etapa terminal, siendo las dimensiones de salud mental y emocional del cuidador las más afectadas, reportando también un aumento en la ansiedad y la depresión, como consecuencia de la falta de control de la situación ${ }^{12,26,27}$. Este hallazgo es importante cuando se contrasta con los resultados de este estudio, donde los cuidadores reportaron niveles medios de calidad de vida y sus dimensiones, valores que pueden explicarse por aspectos propios del paciente como los niveles de funcionalidad y también por características del cuidador como contar con apoyo familiar y un cuidador secundario específico. Sin embargo, la dimensión de bienestar espiritual es la que muestra el puntaje más bajo, lo que podría indicar el impacto espiritual y la necesidad de auto trascendencia y encontrar sentido a la experiencia que se ha documentado en otros estudios ${ }^{28}$, esta afirmación se ve respaldada por el alto compromiso religioso que los participantes reportaron. En otros estudios se ha documentado la necesidad de conexión con un ser supremo para mejorar la espiritualidad ${ }^{28}$.

En cuanto a las correlaciones derivadas del análisis estadístico, es interesante la magnitud moderada a fuerte de las relaciones entre las variables, siendo así, se encuentra que la sobrecarga del cuidado tiene una correlación significativa moderada inversa con la calidad de vida del cuidador, lo cual esta acorde con otros estudios $7,11,28$ en los que cuidadores reportan peores niveles de calidad de vida cuando la percepción
Es interesante la magnitud moderada a fuerte de las relaciones entre las variables, siendo así, se encuentra que la sobrecarga del cuidado tiene una correlación significativa moderada inversa con la calidad de vida del cuidador, lo cual esta acorde con otros estudios $7,11,28$ de sobrecarga es mayor, lo que genera afectaciones en especial en la dimensión de bienestar psicológico. Fueron varias las correlaciones que se obtuvieron, las cuales se pueden explicar desde el comportamiento del cuidador, el estado del paciente y el soporte social con el que cuenta.

Para la dimensión de bienestar físico de la calidad de vida, se presentaron correlaciones significativas débiles e inversas con el impacto del cuidado, la dimensión interpersonal y el total de la sobrecarga del cuidado, esto es coherente cuando se analiza que la funcionalidad de la persona en cuidado paliativo juega un papel primordial en la sobrecarga del cuidador, de manera que el avance de la enfermedad y la cercanía a la muerte, hacen que el cuidador deba suplir más actividades básicas de la vida diaria del paciente y manejar los síntomas de este, lo que genera cansancio e impacto de tipo físico ${ }^{7,23,24}$. Adicional a esto, hay una dedicación constante al cuidado que impacta en el desempeño de otros roles y genera desgaste físico en el cuidador ${ }^{29}$. 
La dimensión psicológica de la calidad de vida también mostró correlaciones significativas débiles inversas con el impacto del cuidado, la dimensión interpersonal y el total de la sobrecarga del cuidado. Como se ha descrito, desde lo psicológico, el impacto que tiene asumir el cuidado de una persona cuya trayectoria de la enfermedad tienen cambios constantes e imprevistos y la fluctuación de los síntomas de las personas con cáncer en cuidado paliativo, conlleva a una falta de control de la situación por parte del cuidador, generando alteraciones en su estabilidad emocional y su salud mental ${ }^{20,26}$. Se ha
Desde lo psicológico, el impacto que tiene asumir el cuidado de una persona cuya trayectoria de la enfermedad tienen cambios constantes e imprevistos y la fluctuación de los síntomas de las personas con cáncer en cuidado paliativo, conlleva a una falta de control de la situación por parte del cuidador, generando alteraciones en su estabilidad emocional y su salud mental ${ }^{20,26}$. documentado además, que son múltiples los cambios a los que se enfrenta el cuidador familiar, con complejas interacciones con otros individuos significativos dentro de su rol, como son el receptor del cuidado, la familia y el equipo de salud, lo que puede generar una sobrecarga de las labores a realizar en el día a día, que finalmente impacta en resultados como el deterioro de su calidad de vida ${ }^{30}$, sobrecarga del cuidado ${ }^{31}$, estrés, ansiedad, soledad y depresión ${ }^{32}$.

Aunque el estudio realizado por Gómez et al. ${ }^{33}$, se enfoca a cuidadores familiares de personas con discapacidad, los resultados descritos sobre las afectaciones en el bienestar psicológico que produce la sobrecarga del cuidado se pueden extrapolar al cuidador del paciente en cuidados paliativos. Dicho estudio describió una influencia del género del cuidador, la fragilidad en redes de apoyo y relaciones interpersonales, y la multiplicidad de funciones a cargo sobre los niveles de ansiedad y depresión del cuidador. Dentro de estos resultados también se evidenció que un $80 \%$ de la muestra presentó rasgos de ansiedad y un $82 \%$ de depresión, sumado a que un $69 \%$ de los cuidadores experimentó sobrecarga en su labor. En consecuencia, la literatura parece exponer que asumir el rol de cuidador familiar implica no solo cambios en la dinámica personal y familiar sino también en la estabilidad emocional y psicológica de este.

En relación con la dimensión de preocupaciones sociales de la calidad de vida, esta se asoció de manera fuerte e inversa con el impacto del cuidado, la dimensión interpersonal y el total de la sobrecarga del cuidado. Estos resultados exponen la necesidad de fortalecer las redes de apoyo y favorecer el desarrollo de otras relaciones interpersonales a parte de las que se tiene con la persona enferma e incluso con la misma familia, con el fin de emplear parte de su tiempo en otras actividades orientadas al autocuidado y en consecuencia mejorar su calidad de vida. Estas afirmaciones, las apoya Vargas y Pinto $^{34}$ que describieron la calidad de vida de cuidadores de personas con Alzheimer, y reportaron que el $66.2 \%$ de estos presentaban alteraciones en sus relaciones personales y el $65 \%$ consideraba que tenía problemas de aislamiento social. Asimismo, algunas investigaciones han documentando que el apoyo social aumenta el bienestar del cuidador ${ }^{35}$, favorece un cambio positivo de las emociones y sentimientos negativos que están presentes en la labor del cuidado, es decir, que les permite resignificar la experiencia ${ }^{36} \mathrm{y}$ ayudar al manejo del estrés, la ansiedad y la depresión causada por el desempeño del rol ${ }^{27}$.

Los resultados de este estudio describen el impacto de la sobrecarga y las relaciones interpersonales dentro de la vida social del cuidador, aunque los participantes refirieron contar con el apoyo familiar y además de un cuidador secundario en su mayoría, el dedicar muchas horas al día al cuidado de la persona en cuidado paliativo deja poco tiempo para el establecimiento de relaciones de amistad o momentos de descanso, por lo que si un cuidador no tiene estos espacios de respiro en el desarrollo su rol, hay una peor percepción de calidad de vida, por encontrarse agobiado con el cuidado de su familiar ${ }^{13,30}$. 
Por otra parte, la dimensión espiritual de la calidad de vida presentó correlaciones inversas con el impacto del cuidado y el total de la sobrecarga del cuidado. Esto puede argumentarse desde la misma vivencia del cuidado de una persona que esta cerca de la muerte, ya que esto genera cuestionamientos personales en el cuidador, lo lleva a repensar incluso en su propias creencias religiosas ${ }^{37}$. Sin embargo, desde otro punto de vista, el comprender el sufrimiento del otro y los significados que esta experiencia les permite fortalecer la dimensión espiritual del cuidador $^{38}$.

Finalmente, el modelo de regresión derivado del análisis muestra que la sobrecarga del cuidador es predictor inverso de sus niveles de calidad de vida, lo cual concuerda con otros estudios similares en cuidadores de personas con enfermedades crónicas. Para la sobrecarga del cuidado, Perpiña et al. ${ }^{11}$ encontraron en su modelo de regresión que aspectos como la ansiedad y la depresión, que podrían estar relacionados con el bienestar psicológico del cuidador, fueron variables que predecían la percepción de la sobrecarga del cuidado $\left(r^{2}=0.52\right)$. Así, los resultados de esta investigación junto con los de Achury et al..$^{39}$, indican que con mayor sobrecarga, peor es la calidad de vida percibida, donde se afectan principalmente la esfera emocional y mental del cuidador, en especial por brindar cuidado a un familiar que se acerca a la muerte, momento donde se empieza a generar el cierre de ciclos con la persona cuidada y un duelo anticipado. Los hallazgos de Ferraz dos Anjos et al. ${ }^{40}$ ratifican los resultados de este estudio, en este caso, los investigadores encontraron en el modelo de regresión que el grado de dependencia del paciente y la sobrecarga de las actividades del cuidador, fueron variables significativas que explicaban una percepción más baja de calidad de vida de los cuidadores.

El modelo en la presente investigación también presenta que los cuidadores con unión conyugal tienen mejor percepción de calidad de vida, esto puede ser explicado desde los aspectos interpersonales de la sobrecarga del cuidado. Cuando el cuidador tiene una pareja estable, esta puede funcionar como parte del apoyo social y emocional que requiere para ejercer el rol del cuidador, lo cual le permite tolerar mejor el estrés, la ansiedad y la angustia de cuidar ${ }^{20}$; además, los cuidadores emplean parte de su tiempo a la vida en pareja, lo que saca al cuidador de la rutina del cuidado, percibiendo así una mejora en la calidad de vida.

Las implicaciones que los resultados de este estudio tienen para los profesionales de enfermería están relacionadas con la importancia de detectar en los cuidadores un cansancio con su rol, ya que como se ha comprobado, esta sobrecarga influye en su calidad de vida, por esta razón brindar un adecuado soporte social donde se fortalezca el rol del cuidador secundario y donde exista apoyo en el desarrollo de estrategias de afrontamiento, es ideal para mejorar la atención dada al cuidador. En primer lugar, la educación al cuidador permite que tenga un mejor manejo y control de la situación, además de ayudarle a prepararse para la muerte de su familiar. En segundo lugar, el apoyo social es fundamental para mejorar la percepción de calidad de vida, así que el profesional de enfermería debe ayudar al cuidador a fortalecer sus redes familiares y la definición de roles dentro de la dinámica familiar para disminuir la sobrecarga. Por último, de manera interdisciplinar se debe orientar, guiar y acompañar al cuidador en el afrontamiento al final de la vida de su familiar para disminuir el impacto psicológico y emocional en su calidad de vida.

En esta investigación se reconocen algunas limitaciones. En primer lugar, el tamaño de la muestra fue relativamente pequeño, lo que puede reducir la capacidad de generalización de los resultados, a pesar de las correlaciones fuertes encontradas. En segundo lugar, el muestreo intencional no permitió una inclusión aleatoria de los participantes al estudio aspecto que se relaciona con inclusión de variables confusoras. En tercer lugar, debido al tipo de población, fue 
difícil el acceso por el momento de cuidado en que se encontraban los participantes y el estado de salud de su familiar.

\section{Conclusión}

La calidad de vida es multidimensional y se asocia con la sobrecarga del cuidado del cuidador de persona con cáncer en cuidado paliativo, lo cual indica que, a mayor nivel de sobrecarga, menor será el nivel de calidad de vida. Dentro de esta línea, este estudio encontró que el bienestar psicológico y las preocupaciones sociales son las dimensiones más afectadas en estos cuidadores. A pesar de la multidimensionalidad de las variables, se presentó una magnitud de moderada a fuerte en las correlaciones, demostrando que, al mejorar las relaciones interpersonales y el manejo de las situaciones del cuidado, se puede presentar una mejora en los aspectos psicológicos y sociales de la calidad de vida. Este estudio identificó que el tener un estatus conyugal (casado o en unión libre) y la presencia de sobrecarga son predictores de la calidad de vida del cuidador familiar.

Por último, se espera que con los resultados de esta investigación se logren desarrollar más estudios longitudinales alrededor de la calidad de vida, incluyendo otras variables desde el afrontamiento y el apoyo social del cuidador de personas con cáncer en cuidado paliativo. Adicional, es prioritario el avance hacia las propuestas de intervenciones en enfermería e interdisciplinares que disminuyan la sobrecarga de la enfermedad y mejoren la calidad de vida del cuidador.

Conflicto de intereses: los autores no declaran conflicto de intereses.

Financiamiento: Proyecto financiado por la convocatoria Minciencias 752 de 2018.

\section{Referencias}

1. World Health Organization. Noncommunicable Diseases Country Profiles 2018. World Health Organization. Geneva; 2018.

2. Worl Health Organization. Definition of Palliative Care. http://www.who.int/cancer/palliative/definition/en/\#. 2015

3. Pastrana T, De Lima L, Pons JC. Atlas de Cuidados Paliativos de Latinoamérica. Houston; 2013.

4. Garcia-Rueda N, Carvajal Valcarcel A, Saracibar-Razquin M, Arantzamendi Solabarrieta $\mathbf{M}$. The experience of living with advanced-stage cancer: a thematic synthesis of the literature. Eur J Cancer Care. 2016;25(4):551-69. DOI: https://doi.org/10.1111/ecc.12523

5. Arias-Rojas M, Carreño-Moreno S, Chaparro L. Incertidumbre ante la enfermedad crónica. Revisión integrativa. Rev Latinoam Bioética. 2019;36(1):91-104.

DOI: https://doi.org/10.18359/rlbi.3575

6. Butow PN, Price MA, Bell ML, Webb PM, deFazio A, Friedlander M. Caring for women with ovarian cancer in the last year of life: a longitudinal study of caregiver quality of life, distress and unmet needs. Gynecol Oncol. 2014;132(3):690-7.

DOI: https://doi.org/10.1016/j.ygyno.2014.01.002

7. Ullrich A, Ascherfeld L, Marx G, Bokemeyer C, Bergelt C, Oechsle K. Quality of life, psychological burden, needs, and satisfaction during specialized inpatient palliative care in family caregivers of advanced cancer patients. BMC Palliat Care. 2017;16(1):31.

DOI: https://doi.org/10.1186/s12904-017-0206-z 
8. Oechsle K, Ullrich A, Marx G, Benze G, Heine J, Dickel LM, et al. Psychological burden in family caregivers of patients with advanced cancer at initiation of specialist inpatient palliative care. BMC Palliat Care. 2019;18(1):1-14.

DOI: https://doi.org/10.1186/s12904-019-0469-7

9. Moreira de Souza R, Turrini RN. Paciente oncológico terminal: sobrecarga del cuidador. Enfermería Glob. 2011;10(22):1-13. DOI: https://doi.org/10.6018/eglobal.10.2.122771

10.Naoki Y, Matsuda Y, Maeda I, Kamino H, Kozaki Y, Tokoro A, et al. Association between family satisfaction and caregiver burden in cancer patients receiving outreach palliative care at home. Palliat Support Care. 2018;16(3):260-8.

DOI: https://doi.org/10.1017/S1478951517000232.

11.Perpiñá-Galvañ J, Orts-Beneito N, Fernández-Alcántara M, García-Sanjuán S, GarcíaCaro MP, Cabañero-Martínez MJ. Level of burden and health-related quality of life in caregivers of palliative care patients. Int J Environ Res Public Health. 2019;16(23).

DOI: https://doi.org/10.3390/ijerph16234806

12.Coca S, Ramos F, Fernández R. Calidad de vida en los cuidadores familiares de pacientes con enfermedad terminal, bajo un programa de cuidados paliativos domiciliarios. Rev salud pública. 2017;XXI(3):22-34. DOI: https://doi.org/10.31052/1853.1180.v21.n3.17386

13.Antón BS, Meneses MA. Cansancio del rol de cuidador: análisis de sus factores relacionados. REDUCA. 2014;6(3):74-106.

Disponible en: http://revistareduca.es/index.php/reduca-enfermeria/article/view/1708

14.Grove S, Gray J. Understanding Nursing Research. Building an Evidence-Based Practice. 7th editio. Missouri, EEUU.: Elsevier; 2019. p.652

15.Chaparro-Diaz L, Sánchez B, Carrillo-Gonzalez GM. Encuesta de caracterización del cuidado de la diada cuidadorfamiliar - persona con enfermedad crónica. Rev Cienc y Cuid. 2015;11(2):31-45. DOI: https://doi.org/10.22463/17949831.196

16.Barreto-Osorio RV, Campos de Aldana MS, Carrillo-Gonázdlez GM, Coral-Ibarra R, Chaparro-Diaz L, Durádn-Parra M, et al. Entrevista Percepción de Carga del Cuidado de Zarit: pruebas psicométricas para Colombia. Aquichan. 2015;15(3):368-80.

DOI: https://doi.org/10.5294/aqui.2015.15.3.5

17.Albarracín Á, Cerquera A, Pabón D. Escala de sobrecarga del cuidador zarit: estructura factorial en cuidadores informales de Bucaramanga. Rev Psicol Univ Antioquia. 2016;8(2):8799. DOI: https://doi.org/10.17533/udea.rpsua.v8n2a06

18. Barrera OL, Carrillo GM, Chaparro DL, Sánchez HB, Vargas RE, Carreño SP. Validez de constructo y confiabilidad del instrumento calidad de vida versión familiar en español. Enfermería Glob. 2015;14(37):227-38. DOI: https://doi.org/10.6018/eglobal.14.1.185111

19.Gray JR, Grove SK, Sutherland S. The practice of nursing research: appraisal, synthesis, and generation of evidence. 8th ed. St. Louis, Missouri: Elsevier; 2016.

20.Rizo VA, Molina LM, Milián MN, Pagán MP, Machado GJ. Caracterización del cuidador primario de enfermo oncológico en estado avanzado. Rev Cuba Med Gen Integr. 2016;32(3):1-13.

21.Flores M, Fuentes H, González G, Meza I, Cervantes G, Valle M. Características principales del cuidador primario informal de adultos mayores hospitalizados. Nure Inv. 2017;14(88):1-16.

22.Lara PG, Pedraza AA, Blanco LL. Perfil del cuidador: sobrecarga y apoyo familiar e institucional del cuidador primario en el primer nivel de atención. Rev Espec MédicoQuirúrgicas. 2008;13(4):159-66.

23.Barragán BJ. Necesidades de cuidado de la diada cuidador-persona: Expectativa de cambio en intervenciones de enfermería. Rev Cuid. 2014;5(2).

DOI: http://dx.doi.org/10.15649/cuidarte.v5i2.87 
24.Sklenarova H, Krümpelmann A, Haun MW, Friederich HC, Huber J, Thomas M, et al. When do we need to care about the caregiver? Supportive care needs, anxiety, and depression among informal caregivers of patients with cancer and cancer survivors. Cancer. 2015;121(9):1513-9. DOI: https://doi.org/10.1002/cncr.29223

25.Vahidi M, Mahdavi N, Asghari E, Ebrahimi H, Eivazi Ziaei J, Hosseinzadeh M, et al. Other side of breast cancer: factors associated with caregiver burden. Asian Nurs Res. 2016;10(3):2016. DOI: https://doi.org/10.1016/j.anr.2016.06.002

26.Götze H, Brähler E, Gansera L, Schnabel A, Gottschalk-Fleischer A, Köhler N. Anxiety, depression and quality of life in family caregivers of palliative cancer patients during home care and after the patient's death. Eur J Cancer Care. 2018;27(2):1-8.

DOI: https://doi.org/10.1111/ecc.12606

27.Domínguez JA, Ruíz M, Gómez I, Gallego E, Valero J, Izquierdo MT. Ansiedad y depresión en cuidadores de pacientes dependientes. Semergen. 2012;38(1):16-23.

DOI: https://doi.org/10.1016/j.semerg.2011.05.013

28.Krug K, Miksch A, Peters-Klimm F, Engeser P, Szecsenyi J. Correlation between patient quality of life in palliative care and burden of their family caregivers: A prospective observational cohort study. BMC Palliat Care. 2016;15(1):1-8.

DOI: http://dx.doi.org/10.1186/s12904-016-0082-y

29.Toribio-Díaz ME, Medrano-Martínez V, Moltó-Jordá JM, Beltrán-Blasco I. Red de cuidadores informales de los pacientes con demencia en la provincia de Alicante, descripción de sus características. Neurologia. 2013;28(2):95-102.

DOI: http://dx.doi.org/10.1016/j.nrl.2012.03.010

30.Ávila-Toscano JH, Vergara Mercado M. Calidad de vida en cuidadores informales de personas con enfermedades crónicas. Aquichan. 2014;14(3):417-29.

DOI: https://doi.org/10.5294/aqui.2014.14.3.11

31.Carreño S, Chaparro L. Agrupaciones de cuidadores familiares en Colombia: perfil, habilidad de cuidado y sobrecarga. Pensam Psicológico. 2017;15(1):87-101.

DOI: http://dx.doi.org/10.11144/Javerianacali.PPSI15-1.ACFC

32.Klassen AF, Gulati S, Granek L, Rosenberg-Yunger ZRS, Watt L, Sung L, et al. Understanding the health impact of caregiving: a qualitative study of immigrant parents and single parents of children with cancer. Qual Life Res. 2012;21(9):1595-605.

DOI: https://doi.org/10.1007/s11136-011-0072-8

33.Gómez-Galindo AM, Peñas-Felizzola OLL, Parra-Esquivel El. Caracterización y condiciones de los cuidadores de personas con discapacidad severa en Bogotá. Rev Salud Pública. 2016;18(3):367. DOI: http://dx.doi.org/10.15446/rsap.v18n3.53048

34.Vargas L, Pinto N. Calidad de vida del cuidador familiar y dependencia del paciente con Alzheimer. Av Enferm. 2010;28(1):116-128.

35.Colin Reid R, Stajduhar KI, Chappell NL. The impact of work interferences on family caregiver outcomes. J Appl Gerontol. 2010;29(3):267-89.

DOI: https://doi.org/10.1177/0733464809339591

36.Domínguez Guedea MT, García AO, Rivera Sander MA. Bienestar, Apoyo social y contexto familiar de cuidadores de adultos mayores. Acta Investig Psicológica. 2013;3(1):1018-30.

DOI: https://doi.org/10.1016/S2007-4719(13)70949-9

37.Pérez-García E. Enfermería y necesidades espirituales en el paciente con enfermedad en etapa terminal. Enfermería Cuid Humaniz. 2016;5(2):41-45.

DOI: https://doi.org/10.22235/ech.v5i2.1286

38. Rodrigues GA. La espiritualidad ante la proximidad de la muerte... Enfermería Glob. 2011;10(22):1-10. DOI: https://doi.org/10.4321/S1695-61412011000200019 
39.Achury DM, Pinilla M. La comunicación con la familia del paciente que se encuentra al final de la vida. Enfermería Univ. 2016;13(1):55-60. DOI: https://doi.org/10.1016/j.reu.2015.12.001 40.Ferraz dos AK, Silva de OB, Cruz SV, Nagib BE, Kareny da SJ, de Oliveira SR. Fatores associados à qualidade de vida de cuidadores familiares de idosos. Ciencenferm.2018;24(17):115. DOI: http://dx.doi.org/10.4067/s0717-95532018000100217. 